REPRINTED FROM ANNALS OF OTOLOGY, RHINOLOGY \& LARYNGOLOGY 1987; 96(Suppl 128)

\title{
AUDIOLOGICAL ASSESSMENT OF PROFOUNDLY HEARING-IMPAIRED CHILDREN
}

\author{
P. A. Busby, MA, Dip Aud: R. C. Dowell, BSc, Dip Aud; T. G. Nienhuys, PhD; G. M. Clark, PhD, FRACS
}

Supported by the Lions International Research Fellowship.

The design of an audiological assessment protocol for profoundly hearing-impaired children may be divided into three areas. First, accurate estimation of hearing loss includes the behavioral measures of unaided and aided thresholds and the objective measures of electrocochleography and auditory brain stem response. The reliability of these measures for the accurate diagnosis of a profound to total hearing loss is discussed. Second, speech perception includes the measure of perception in the audition alone, vision alone, and audition plus vision conditions. Test material should include speech features, words, and sentences. Factors influencing the choice of material are the developmental age of the child, the method of educational instruction, speech and language skills, and vocabulary limits. Third, psychophysical properties of residual auditory skills include measures such as frequency, intensity, and duration difference limens. These skills may be compared to those elicited through other sensory chamels, such as visual and tactile. Other important factors that should be considered are the psychological well-being of the child and family, family motivations and expectations, and educational requirements.

The audiological assessment of the profoundly hearingimpaired child is becoming increasingly important in the selection of the most appropriate sensory devices. These devices include high gain hearing aids, tactile and electrotactile aids, and cochlear implants. This paper proposes three areas of investigation that are important in the audiological assessment protocol for device selection.

The first area of investigation concerns the accurate estimation of hearing thresholds. Behavioral pure tone audiometry is usually less reliable for children below 3 to 4 years of age than for children above this age. Northern and Downs ${ }^{1}$ have suggested that responses for the younger children may be approximately $25 \mathrm{~dB}$ above true thresholds. The sophistication of the procedure and the ability of the child to respond are dependent on a number of factors. These include the developmental and chronological age, interest in the procedure, prior auditory experience, test environment, stimulus characteristics, and status of motor and perceptual abilities. ${ }^{2}$ Also important in cases of acquired losses are the years of profound loss and the age of the child at the onset of the impairment. Failure to consider these factors may result in inaccurate behavioral threshold estimations before sensory device fitting. For example, it has been reported during postoperative assessment that some preschool-aged children provided with single-channel cochlear implants were able to respond more consistently to acoustic stimulation in the nonimplanted ear fitted with a hearing aid than they were preoperatively. ${ }^{3}$ This may suggest that, preoperatively, insufficient attention was directed toward the elicitation of consistent and realistic estimations of thresholds in these children.

Objective estimations of hearing thresholds should include electrocochleography (ECoG) and auditory brain stem response (ABR) audiometry. Both have some limitations for the full assessment of children. First, the characteristics of stimuli commonly used, for example broad band clicks, do not provide information concerning discrete frequencies usually assessed in behavioral audiometry. Second. $\mathrm{ABR}$ or $\mathrm{ECoG}$ electrical potentials may be approximately $20 \mathrm{~dB}$ above the behavioral thresholds because of difficulties in identifying the response waves. ${ }^{4}$ Rickards ${ }^{5}$ has demonstrated frequency-specific electrical potentials to amplitude-modulated and frequency-modulated tones; thus an estimation of discrete frequency thresholds may be obtained. Nowosielski and Redhead ${ }^{\dagger}$ have demonstrated an effective technique for the use of high intensity signals, up to $115 \mathrm{~dB}$ hearing threshold level (HTL), in ECoG recordings. A piezoelectric tweeter housed in an electrostatically shielded cover and a different method of needle anchorage have been used to overcome some signal and noise difficulties usually associated with high intensity stimulation.

The second area for investigation is concerned with the measurement of the contributions of the acoustic signal to speech perception. This is particularly important for the estimation of the communication handicap imposed by a profound loss. For this purpose, stimuli should be presented in the audition alone, vision alone, and audition plus vision conditions because these represent the more common speech signal channels available to the hearing-impaired child. ${ }^{7}$ The contributions of acoustic and visual speech signals have been frequently assessed by the use of these three conditions. For example, in the perception of vowels by hearing-impaired children it has been demonstrated that some patients were able effectively to combine acoustic and visual parameters, whereas others were not. ${ }^{8}$ In respect to speech perception in the audition alone condition, some profoundly hearing-impaired children can perceive a significant proportion of phonological features in the speech signal. ${ }^{9}$ Boothroyd ${ }^{10}$ has also suggested that children with hearing thresholds up to $110 \mathrm{~dB}$ HTL have useful hearing for both speech perception and production when used in an effective training program. The training program itself is an important contributor to the development of these skills with the sensory device. ${ }^{11.12}$

A large selection of stimuli is available for the assessment of speech perception by hearing-impaired children. For example, consonant and vowel confusion studies provide information concerning phoneme discrimination. The monosyllable-spondee-trochee (MST) discrimination test ${ }^{13}$ measures syllable pattern categorization and within-pattern discrimination. Bamford-Kowal-Bench sentences ${ }^{14}$ provide a measure that reflects on the communicative perceptual skills of a child. However, the significance of the results is often limited by the material used. For example, withinpattern discrimination for the MST test may be dependent on pattern categorization skills. Vowel and consonant confusion studies may not necessarily reflect on the communicative perceptual skills of the hearing-impaired child. The choice of materials is also governed by the same factors that influence the estimation of behavioral thresholds. In addition, other factors should also be considered. First, the speech and language skills of the child would influence the choice of stimuli and the test administration. Second, vocabulary size may restrict the choice of test items because all stimuli should be within the limits of the child. Third, the method of educational instruction is important because experience with acoustic and visual speech signals influences the child's abilities to perform speech tests in these 
modalities.

The third area for investigation is concerned with the measurement of the psychophysical properties of residual auditory skills. For instance, frequency difference limens may be used to suggest that the behavioral thresholds reflect tactile rather than auditory responses. ${ }^{15}$ Other properties concerned with intensity and temporal integration may also be included; for example, amplitude difference limens for the former and gap detection ${ }^{16}$ and numerosity skills ${ }^{17}$ for the latter. These can be studied in respect to the relationship between speech perception and psychophysical properties $^{18}$ and in relation to performance in other modalities, such as tactile and visual.

The data collected by these assessments would provide information that assists in the selection of the most appropriate sensory device for the profoundly hearing-impaired child. Also, information for the effective auditory management of the child in an educational training program would be available. Other factors relevant to the implementation of auditory management would include the psychological and neurological status of the child, family motivations and expectations with the sensory device, and the speech and language requirements of the child within the educational setting.

Careful audiological assessment and management over a realistic time period would provide an indication of the speech perception skills of the child and indicate the usefulness of the sensory device fitted. The change to other devices, such as different hearing aids, tactile or electrotactile aids, or cochlear implants could be suggested after effective assessments with the fitted device. Family and teachers would also be more informed of the device options available to the profoundly hearing-impaired child as a consequence of the interpretation of data from these assessments.

\section{REFERENCES}

1. Northern JL. Downs MP. Hearing in children. Baltimore: Williams and Wilkins, 1978.

2. Hodgson WR. Testing infants and young children. In: Katz J, ed. Handbook of clinical audiology. Baltinore: Willians and Wilkins, 1978:397-409.

3. House WF, Eisenberg IS. The cochlear implant in preschool- aged children. Acta Otolaryngol (Stockh) r983;95:6i32-8.

4. Davis H. Brainstem and other responses in electrical response audiometry. Ann Otol Rhinol Laryngol 1976;85:3-14.

5. Rickards FW. Auditory steady-state evoked potentials in humans to anplitude modulated tones [Dissertation]. Melbourne, Australia: University of Melbourne, I983.

6. Nowosiclski J, Redhead J. An improved technique for simultaneous recording of action potential and auditory brainstem responses. Br J Audiol 1985:19:13-8.

7. Ling D. Specch and the hearing-impaired child: theory and practice. Washington, DC: AG Bell Assoc Deaf, 1976.

8. Busby PA, Tong YC, Clark GM. Underlying dinrensions and individual differences in auditorv, sisual, and anditory-visual vowel perception hy hearing-impaired children. J Acoust Soc Am 1984;75:1858-65.

9. Boothroyd A. Auditory perception of spech contrasts by suljects with sensorineural hearing loss. I Speech Hear Res 1984: 27:134-44.

10. Boothroyd A. Influence of residual hearing on speech perception and spech production by hearing inpaired children. SARP No. 26. Northampton, Mass: Clarke School for Deaf, 1976.

11. Simmons FB. Cochlear implants in young children: some dilemmas. Ear Hear 1985;6:61-3.

12. Pickett JM, McFarland W. Auditory implants and tactile aids for the profoundly deaf. J Specch Hear Res 1985:28: 134-50.

13. Erber NP, Alencewicz CM. Audiologic evaluation of deaf children. J Speech Hear Dis 1976:41:256-67.

14. Bench J, Bamford J. cds. Speech-hearing tests and the spoken language of hearing-impaired children. London: Academic Prcss, 1979 .

15. Risberg A, Agelfors E, Boberg G. Measurement of frequency-discrimination ability of severely and profoundly hearingimpaired children. Speech Transmission I aboratory-Quarterly Progress and Status Report, Royal Institute of Technology, Sweden, $1975 ;$ no. $2-3: 40-8$.

16. Tyler RS, Summerfield Q, Wood EJ, Fernandes MA. Psychoacoustic and phonctic temporal processing in normal and hearingimpaired listeners. J Acoust Soc Am 1982:72:740-52.

17. Lechelt EC. Temporal numerosity discrimination: intermodal comparisons revisited. Br J Psychol 1975:66:101-8.

18. Dreschler WA. Plonp R. Relation between psychophysical data and speech perception for hearing-impaired subjects. I. J Acoust Soc Am 1980;68:1608-15. 


\section{University Library}

\section{- M M I N E R VA A gateway to Melbourne's research publications}

Minerva Access is the Institutional Repository of The University of Melbourne

Author/s:

Busby, P. A.;Dowell, R. C.;Nienhuys, T. G.;Clark, Graeme M.

Title:

Audiological assessment of profoundly hearing-impaired children

Date:

1987

Citation:

Busby, P. A., Dowell, R. C., Nienhuys, T. G., \& Clark, G. M. (1987). Audiological assessment of profoundly hearing-impaired children. Annals of Otology, Rhinology \& Laryngology, 96(suppl.128), 85-86.

Persistent Link:

http://hdl.handle.net/11343/27240 